\title{
On Defense Strength of Blocking Defeaters in Admissible Sets
}

\author{
Diego C. Martínez, Alejandro J. García, and Guillermo R. Simari \\ Comisión Nacional de Investigaciones Científicas y Técnicas - CONICET \\ Artificial Intelligence Research and Development Laboratory (LIDIA) \\ Department of Computer Science and Engineering, Universidad Nacional del Sur \\ Av. Alem 1253 - (8000) Bahía Blanca - Bs. As. - Argentina \\ $\{$ dcm, ajg, grs\}@cs.uns.edu. ar
}

\begin{abstract}
Extended argumentation framework is a formalism where defeat relations are determined by establishing a preference between arguments involved in symmetric conflicts. This process possibly leads to blocking situations, where conflicting arguments are found to be incomparable or equivalent in strength. In this work we introduce new argumentation semantics for extended frameworks, by taking into account the strength of an argument defense. Each of these new admissibility notions relies on the presence of blocking defeaters as argument defenders.
\end{abstract}

\section{Introduction}

Argumentation has become an important subject of research in Artificial Intelligence and it is also of interest in several disciplines, such as Logic, Philosophy and Communication Theory. This wide range of attention is due to the constant presence of argumentation in many activities, most of them related to social interactions between humans, as in civil debates, legal reasoning or every day dialogues. Basically, an argument is a piece of reasoning that supports a claim from certain evidence. The tenability of this claim must be confirmed by analyzing other arguments for and against such a claim. In formal systems of defeasible argumentation, a claim will be accepted if there exists an argument that supports it, and this argument is acceptable according to an analysis between it and its counterarguments. After this dialectical analysis is performed over the set of arguments in the system, some of them will be acceptable, justified or warranted arguments, while others will be not. The study of the acceptability of arguments is the search for rationally based positions of acceptance in a given scenario of arguments and their relationships. It is one of the main concerns in Argumentation Theory.

Abstract argumentation systems [12344 are formalisms for argumentation where some components remain unspecified, being the structure of an argument the main abstraction. In this kind of system, the emphasis is put on the semantic notion of finding the set of accepted arguments. Most of them are based on the single abstract concept of attack represented as an abstract relation, and extensions are defined as sets of possibly accepted arguments. For two arguments

Z. Zhang and J. Siekmann (Eds.): KSEM 2007, LNAI 4798, pp. $140+152,2007$.
(C) Springer-Verlag Berlin Heidelberg 2007 
$\mathcal{A}$ and $\mathcal{B}$, if $(\mathcal{A}, \mathcal{B})$ is in the attack relation, then the acceptance of $\mathcal{B}$ is conditioned by the acceptance of $\mathcal{A}$, but not the other way around. It is said that argument $\mathcal{A}$ attacks $\mathcal{B}$, and it implies a priority between conflicting arguments. It is widely understood that this priority is related to the argument strengths. Several frameworks do include an argument order $3 \mid 5,6$, although this order is used at another level, as the classic attack relation is kept.

In 78 an extended abstract argumentation framework is introduced, where two kinds of defeat relations are present. These relations are obtained by applying a preference criterion between conflictive arguments. The conflict relation is kept in its most basic, abstract form: two arguments are in conflict simply if both arguments cannot be accepted simultaneously. The preference criterion subsumes any evaluation on arguments and it is used to determine the direction of the attack. This argument comparison, however, is not always succesful and therefore attacks, as known in classic frameworks, are no longer valid. For example, consider the following argument, called $N S_{1}$ :

$N S_{1}$ : Buy new ski tables, because they are specially shaped to improve turns.

The claim of $N S_{1}$ is that new skis must be bought, for a reason about skiing performance. Now suppose a new argument, called $O S$ is introduced

OS: Buy used old ski tables, because they are cheap and your skills are good enough to face any turn.

This argument exposes two reasons for buying old skis. Clearly, $O S$ is in conflict with $N S_{1}$ as both arguments cannot be accepted altogether: we buy new skis, or we buy old ones. Given this dichotomy, both argument must be compared to each other. The claim of argument $O S$ is supported by more reasons than $N S_{1}$, and one of them is also about skiing performance. Therefore, it is valid to state that $O S$ is preferable to $N S_{1}$, and then the former became a proper defeater of the latter. This is the strongest form of attack. Now consider two additional arguments, called $N S_{2}$ and $N S_{3}$ :

$N S_{2}$ : Buy new ski tables, as they are not expensive, and old used ski tables are not helping to improve your skills.

$N_{3}$ : Buy new ski tables, because the colors and graphic styles are cool.

Both arguments are supporting the same claim ("Buy new ski tables"), and therefore they are in conflict with $O S$. Again, a comparison is needed. The argument $N S_{2}$ states that new skis are not expensive and they can improve skills. As it refers to price and performance, the information exposed in $N S_{2}$ is as strong as the one exposed in $O S$. In fact, both arguments can be considered equally strong when supporting its claims. Therefore, $N S_{2}$ and $O S$ are blocking each other, and then both arguments are said to be blocking defeaters. On the other hand, $N S_{3}$ is based on style taste to support its claim. No reference to the money invested or to personal skills is made. Although they are in conflict, $N S_{3}$ and $O S$ cannot be compared to each other. Arguments $N S_{3}$ and $O S$ are also blocking defeaters, but of different nature, as they are incomparable. 
Extended argumentation frameworks $(E A F)$ are suitable to model situations like above. In [8] a simple semantics based on suppression of arguments is presented, and in 9] the well-formed structure of argumentation lines is introduced. These are two characterizations of unique sets of accepted arguments, according to specific dialectical interpretations. However, under the classic acceptability notions [1] in abstract frameworks it is possible to have several alternative sets of acceptance (extensions) for a given argument scenario. This is a common outcome of attack cycles $\left(\mathcal{A}_{1}\right.$ is attacked by an argument $\mathcal{A}_{2}$, that is attacked by $\mathcal{A}_{3}, \ldots$, attacked by $\mathcal{A}_{n}$, which in turn attacks $\left.\mathcal{A}_{1}\right)$. In extended abstract frameworks this is also present: every argument in the ski example is defeated by another argument. In this case, when searching for accepted arguments it is not easy to find a starting point, as everyone has a defeater. Therefore, several extensions arises. Using extended frameworks, however, it is more clear how defeat occurs regarding to argument strength.

In this work we explore new semantic considerations based on the quality of a defense. In the previous example, arguments $N S_{2}$ and $N S_{3}$ are defeaters of $O S$, which is a defeater of $N S_{1}$. Therefore, $N S_{1}$ is said to be defended by $N S_{2}$ and $N S_{3}$. The ideal defense is achieved by stronger arguments, but in this case a defender is considered as strong as $O S$, and the other is actually incomparable. This scenario leads to several argument extensions. However, in extended abstract frameworks the strength of defenses is a pathway to compare extensions, in a search for a position of acceptance following rational criteria. The fact that arguments play the role of defenders with different strengths, and how it can be used to define new extensions, is the motivation of this work.

This paper is organized as follows. In the next section, the extended argumentation frameworks are formally introduced. In Section 3 a simple notion of admissibility regarding blocked defeaters is presented. In Section 4 and Section 5 new admissibility sets are characterized taking the strength of defense into account. Finally, the conclusions and future work are presented in Section 6 .

\section{Extended Argumentation Framework}

In our extended argumentation framework three relations are considered: conflict, subargument and preference between arguments. The definition follows:

Definition 1. An extended abstract argumentation framework ( $E A F$ ) is a quartet $\Phi=\langle A R, \sqsubseteq, \mathbf{C}, \mathbf{R}\rangle$, where $A R$ is a finite set of arguments, and $\sqsubseteq, \mathbf{C}$ and $\mathbf{R}$ are binary relations over $A R$ denoting respectively subarguments, conflicts and preferences between arguments.

Arguments are abstract entities, as in 11, that will be denoted using calligraphic uppercase letters, possibly with indexes. In this work, the subargument relation is not relevant for the topic addressed. Basically, it is used to model the fact that arguments may include inner pieces of reasoning that can be considered arguments by itself, and it is of special interest in dialectical studies [9]. Hence, unless explicitly specified, in the rest of the paper $\sqsubseteq=\emptyset$. The conflict relation 
C states the incompatibility of acceptance between arguments. Given a set of arguments $S$, an argument $\mathcal{A} \in S$ is said to be in conflict in $S$ if there is an argument $\mathcal{B} \in S$ such that $\{\mathcal{A}, \mathcal{B}\} \in \mathbf{C}$. The relation $\mathbf{R}$ is introduced in the framework and it will be used to evaluate arguments, modeling a preference criterion based on a measure of strength.

Definition 2. Given a set of arguments $A R$, an argument comparison criterion $\mathbf{R}$ is a binary relation on $A R$. If $\mathcal{A} \mathbf{R} \mathcal{B}$ but not $\mathcal{B} \mathbf{R} \mathcal{A}$ then $\mathcal{A}$ is strictly preferred to $\mathcal{B}$, denoted $\mathcal{A} \succ \mathcal{B}$. If $\mathcal{A} \mathbf{R} \mathcal{B}$ and $\mathcal{B} \mathbf{R} \mathcal{A}$ then $\mathcal{A}$ and $\mathcal{B}$ are indifferent arguments with equal relative preference, denoted $\mathcal{A} \equiv \mathcal{B}$. If neither $\mathcal{A} \mathbf{R} \mathcal{B}$ or $\mathcal{B} \mathbf{R} \mathcal{A}$ then $\mathcal{A}$ and $\mathcal{B}$ are incomparable arguments, denoted $\mathcal{A} \bowtie \mathcal{B}$.

For two arguments $\mathcal{A}$ and $\mathcal{B}$ in $A R$, such that the pair $\{\mathcal{A}, \mathcal{B}\}$ belongs to $\mathbf{C}$ the relation $\mathbf{R}$ is considered. In order to elucidate conflicts, the participant arguments must be compared. Depending on the preference order, two notions of argument defeat are derived.

Definition 3. Let $\Phi=\langle A R, \sqsubseteq, \mathbf{C}, \mathbf{R}\rangle$ be an $E A F$ and let $\mathcal{A}$ and $\mathcal{B}$ be two arguments such that $(\mathcal{A}, \mathcal{B}) \in \mathbf{C}$. If $\mathcal{A} \succ \mathcal{B}$ then it is said that $\mathcal{A}$ is a proper defeater of $\mathcal{B}$. If $\mathcal{A} \equiv \mathcal{B}$ or $\mathcal{A} \bowtie \mathcal{B}$, it is said that $\mathcal{A}$ is a blocking defeater of $\mathcal{B}$, and viceversa. An argument $\mathcal{B}$ is said to be a defeater of an argument $\mathcal{A}$ if $\mathcal{B}$ is a blocking or a proper defeater of $\mathcal{A}$.

Example 1. Let $\Phi_{1}=\langle A R, \sqsubseteq, \mathbf{C}, \mathbf{R}\rangle$ be an $E A F$ where $A R=\{\mathcal{A}, \mathcal{B}, \mathcal{C}, \mathcal{D}, \mathcal{E}\}$, $\sqsubseteq=\emptyset, \mathbf{C}=\{\{\mathcal{A}, \mathcal{B}\},\{\mathcal{B}, \mathcal{C}\},\{\mathcal{C}, \mathcal{D}\}\},\{\mathcal{C}, \mathcal{E}\}\}$ and $\mathcal{A} \succ \mathcal{B}, \mathcal{B} \succ \mathcal{C}, \mathcal{E} \bowtie \mathcal{C}, \mathcal{C} \equiv \mathcal{D}$.

Extended abstract frameworks can also be depicted as graphs, with different types of arcs, called EAF-graphs. We represent arguments as black triangles. An arrow $(\rightarrow)$ is used to denote proper defeaters. A double-pointed straight arrow ( $\longleftrightarrow$ ) connects blocking defeaters considered equivalent in strength, and a double-pointed zig-zag arrow ( $\leadsto$ ) connects incomparable blocking defeaters. In Figure 1, the framework $\Phi_{\mathbb{1}}$ is shown. Argument $\mathcal{A}$ is a proper defeater of $\mathcal{B}$. Argument $\mathcal{B}$ is a proper defeater of $\mathcal{C}$, and $\mathcal{E}$ is an incomparable blocking defeater of $\mathcal{C}$ and viceversa. Argument $\mathcal{D}$ and $\mathcal{C}$ are blocking defeaters being equivalent in strength.

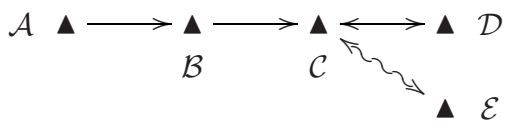

Fig. 1. EAF-graph of framework $\Phi_{1}$

Example 2. The EAF of the introductory example about buying ski tables is $\Phi_{s k i}=\langle A R, \sqsubseteq, \mathbf{C}, \mathbf{R}\rangle$ where $A R=\left\{N S_{1}, O S, N S_{2}, N S_{3}\right\}, \sqsubseteq=\emptyset, \mathbf{C}=\left\{\left\{N S_{1}, O S\right\},\left\{O S, N S_{2}\right\},\left\{O S, N S_{3}\right\}\right\}$ and $O S \succ N S_{1}, O S \equiv N S_{2}, O S \bowtie N S_{3}$. The framework is shown in Figure 2.

The central element of attention in this work is blocking defeat. This kind of defeat arises when no strict preference is established between conflictive arguments. 


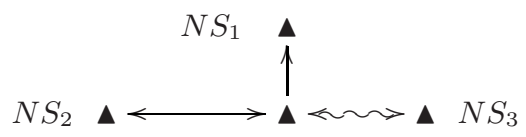

$O S$

Fig. 2. EAF-graph of framework $\Phi_{s k i}$

Even then, blocked arguments are able to defend other arguments, although this defense is achieved with different strenght, as in Example 2. When this happens, a good position to be adopted is the identification of good and bad defenses. In the next section, we present new considerations about acceptability [1] regarding blocking defeaters.

\section{Admissibility}

As said before, argumentation semantics is about argument classification through several rational positions of acceptance. A central notion in most argument extensions is acceptability. A very simple definition of acceptability in extended abstract frameworks is as follows.

Definition 4. Let $\Phi=\langle A R, \sqsubseteq, \mathbf{C}, \mathbf{R}\rangle$ be an EAF. An argument $\mathcal{A} \in A R$ is acceptable with respect to a set of arguments $S \subseteq A R$ if and only if every defeater $\mathcal{B}$ of $\mathcal{A}$ has a defeater in $S$.
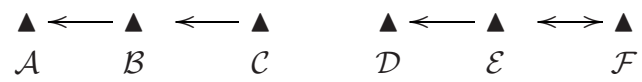

Fig. 3. Simple extended abstract framework

Defeaters mentioned in Definition 4 may be either proper or blocking ones. In the Figure 3, argument $\mathcal{A}$ is acceptable with respect to $\{\mathcal{C}\}$. Argument $\mathcal{C}$ is acceptable with respect to the empty set. Argument $\mathcal{D}$ is acceptable with respect to $\{\mathcal{F}\}, \mathcal{F}$ is acceptable with respect to $\{\mathcal{F}\}$, and so is $\mathcal{E}$ with respect to $\{\mathcal{E}\}$. Note that an argument that is not strictly preferred to another conflicting argument is considered strong enough to stand and defend itself. In Figure $3, \mathcal{F}$ is defending itself against $\mathcal{E}$. Because of this intrinsic property of blocked arguments, a stronger notion of acceptability can be defined, by excluding self-defended arguments.

Definition 5. Let $\Phi=\langle A R$, $, \mathbf{C}, \mathbf{R}\rangle$ be an EAF. An argument $\mathcal{A} \in A R$ is $x$-acceptable with respect to a set of arguments $S \subseteq A R$ if it is acceptable with respect to $S-\{\mathcal{A}\}$.

Clearly, the focus is put in those arguments that need their own defense. In the framework of Figure 3 , argument $\mathcal{D}$ is x-acceptable with respect to $\{\mathcal{F}\}$. 
Argument $\mathcal{F}$ is not x-acceptable with respect to $\{\mathcal{F}\}$, as this argument lacks of other defense than the one provided by itself. In the framework $A F_{\text {ski }}$, argument $N S_{2}$ is x-acceptable with respect to $\left\{N S_{2}, N S_{3}\right\}$.

Following the usual steps in argumentation semantics, the notion of acceptability leads to the notion of admissibility. This requires the definition of conflictfree set of arguments. A set of arguments $S \subseteq A R$ is said to be conflict-free if for all $\mathcal{A}, \mathcal{B} \in S$ it is not the case that $\{\mathcal{A}, \mathcal{B}\} \in \mathbf{C}$.

Definition 6. Let $\Phi=\langle A R$, $, \mathbf{C}, \mathbf{R}\rangle$ be an EAF. A set of arguments $S \subseteq A R$ is said to be admissible if it is conflict-free and every argument in $S$ is acceptable with respect to $S$.

An admissible set is able to defend any argument included in that set. As stated before, some arguments can only be defended by themselves, although they can be defenders of other arguments. Therefore, using the refined version of acceptability of Definition 5, the corresponding admissibility notion is as follows.

Definition 7. Let $\Phi=\langle A R, \sqsubseteq, \mathbf{C}, \mathbf{R}\rangle$ be an EAF. A set of arguments $S \subseteq A R$ is said to be $x$-admissible if it is conflict-free and every argument in $S$ is $x$ acceptable with respect to $S$.

An x-admissible extension $S$ may include a blocked argument $\mathcal{A}$ only if the intrinsic self-defense of $\mathcal{A}$ is superfluous. Every x-admissible extension is admissible by definition. In the framework of Figure 3 , the set $\{\mathcal{D}, \mathcal{F}\}$ is admissible, but not x-admissible. In the framework $\Phi_{s k i}$, the sets $\left\{N S_{1}, N S_{2}\right\}$ and $\left\{N S_{1}, N S_{3}\right\}$ are admissible but not x-admissible. The set $\left\{N S_{1}, N S_{2}, N S_{3}\right\}$ is admissible and x-admissible, as arguments $N S_{2}$ and $N S_{3}$ are defenders of each other.

When blocking defeat is present, $\mathrm{x}$-admissiblity is a stronger notion than classic admissibility. If a set $S$ is admissible but not x-admissible, then at least one argument $\mathcal{A}$ in $S$ has a defender that can only be defended by itself. This may be considered as a sign of weakness of $S$. Consider the framework of Figure 4 The admissible sets are $\emptyset,\{\mathcal{A}\},\{\mathcal{B}\},\{\mathcal{C}\}$ and $\{\mathcal{A}, \mathcal{C}\}$. According to x-admissibilty semantics, only the empty set and $\{\mathcal{A}, \mathcal{C}\}$ are valid extensions.

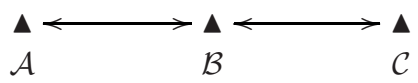

Fig. 4. $\{\mathcal{A}, \mathcal{C}\}$ is x-admissible

An x-admissible set may be a good position of acceptance when trying to avoid arguments that cannot be defended but by themselves. Sometimes, however, these kind of arguments is mandatory for a defense, as in Figure 2 for argument $N S_{1}$. The first step is to distinguish an acceptance set due to defense based on proper-defeaters. In the following section a new semantic notion characterizes a set of accepted arguments only when they benefit from a strictly preferred defense. In Section 5, an argument extension based on the strongest argument defense is introduced. 


\section{Acceptance Through Strongest Defense}

When conflicts and preferences are considered as separate elements in the frameworks, a deeper evaluation of extensions can be considered. Lets analyze the situation depicted in Figure $5(a)$ where $\mathcal{D} \bowtie \mathcal{B}$ and $\mathcal{E} \bowtie \mathcal{C}$.

(a)

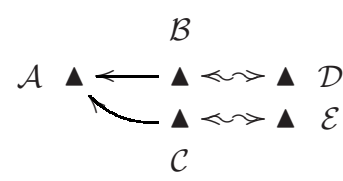

(b)

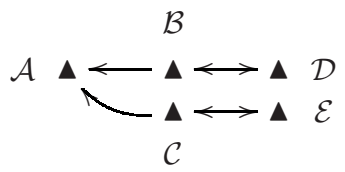

Fig. 5. Argument $\mathcal{A}$ is defended by $\mathcal{D}$ and $\mathcal{E}$

According to Definition 4 , argument $\mathcal{A}$ is acceptable with respect to $\{\mathcal{D}, \mathcal{E}\}$. The set $\{\mathcal{A}, \mathcal{D}, \mathcal{E}\}$ is admissible and it is also a complete extension (in the sense of (1). However, $\{\mathcal{A}, \mathcal{D}, \mathcal{E}\}$ it is not $\mathrm{x}$-admissible, as $\mathcal{D}$ and $\mathcal{E}$ are blocked arguments. In fact, no set including these arguments is $\mathrm{x}$-admissible. Note that the defense of $\mathcal{A}$ is a blockade-based one, that is, it relies on blocking defeat. Now consider the same framework where $\mathcal{D} \equiv \mathcal{B}$ and $\mathcal{E} \equiv \mathcal{C}$, as shown in Figure $5(b)$. There is still a blockade of defeaters of $\mathcal{A}$, but it is due to an equivalence of strength between arguments. Again, $\mathcal{A}$ is defended by $\{\mathcal{D}, \mathcal{E}\}$, but the position is more cohesive. The strenght of arguments can be measured, although only to conclude that it is the same. This is a better defense than blockade by incomparability as before. The quality of a defense determines the quality of an admissible set. For an argument $\mathcal{A}$, being acceptable with respect to a set $S$ does not necessarily means that is strongly defended by $S$. Following the previous example, it is clear that the best defense of $\mathcal{A}$ occurs when $\mathcal{D} \succ \mathcal{B}$ and $\mathcal{E} \succ \mathcal{C}$. In this scenario, $\mathrm{x}$-admissible sets are not a suitable semantics if a credulous position is desired, as the only $\mathrm{x}$-admissible set is the empty set. Thus, the ability to distinguish quality among admissible sets is interesting.

Definition 8. Let $\Phi=\langle A R, \sqsubseteq, \mathbf{C}, \mathbf{R}\rangle$ be an $E A F$. Let $\mathcal{A} \in A R$ be an argument with defeater $\mathcal{B} \in A R$ and let $\mathcal{D} \in A R$ such that $\{\mathcal{B}, \mathcal{D}\} \in \mathbf{C}$. Then argument $\mathcal{A}$ is said to be confirmed by $\mathcal{D}$ against $\mathcal{B}$ if $\mathcal{D} \succ \mathcal{B}$. It is said that $\mathcal{A}$ is sustained by $\mathcal{D}$ against $\mathcal{B}$ if $\mathcal{D} \equiv \mathcal{B}$. Finally, $\mathcal{A}$ is said to be held up by $\mathcal{D}$ against $\mathcal{B}$ if $\mathcal{D} \bowtie \mathcal{B}$. In all cases $\mathcal{D}$ is said to be a defender of $\mathcal{A}$.

Example 3. Consider the EAF of Figure [6. As several blocking situations are present, many cases of defense can be found. For example, argument $\mathcal{A}$ is confirmed by $\mathcal{D}$ against $\mathcal{B}$. It is held up by $\mathcal{F}$ against $\mathcal{B}$, and sustained by $\mathcal{E}$ against $\mathcal{C}$. Argument $\mathcal{C}$, in turn, is confirmed by $\mathcal{B}$ against $\mathcal{A}$. Argument $\mathcal{G}$ is sustained by itself against $\mathcal{H}$.

In the framework of Figure 6 , argument $\mathcal{A}$ is acceptable with respect to $S_{1}=$ $\{\mathcal{E}, \mathcal{F}\}$. It is also acceptable with respect to $S_{2}=\{\mathcal{E}, \mathcal{D}\}$. However, $\mathcal{A}$ is not confirmed by any argument in $S_{1}$, as it is done in $S_{2}$. Therefore, $\{\mathcal{A}\} \cup S_{2}$ may 


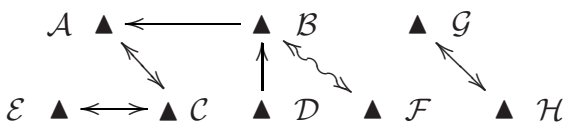

Fig. 6. Argument defense

be considered a stronger admissible set than $\{\mathcal{A}\} \cup S_{1}$. This is reinforced by the fact that $\mathcal{D}$ does not need defense.

Definition 9. Let $\Phi=\langle A R, \sqsubseteq, \mathbf{C}, \mathbf{R}\rangle$ be an $E A F$. An argument $\mathcal{A}$ is strongacceptable with respect to a set of arguments $S \subseteq A R$ if $\mathcal{A}$ is confirmed by an argument in $S$ against every defeater of $\mathcal{A}$.

In the extended framework of Figure 6, argument $\mathcal{F}$ is strong-acceptable with respect to $\{\mathcal{D}\}$. Argument $\mathcal{A}$ is not strong-acceptable with respect to any set, because it cannot be confirmed against $\mathcal{C}$. Argument $\mathcal{D}$ is strong-acceptable with respect to the empty set, as it has no defeaters.

Definition 10. Let $\Phi=\langle A R, \sqsubseteq, \mathbf{C}, \mathbf{R}\rangle$ be an EAF. A set of arguments $S \subseteq A R$ is strong-admissible if every argument in $S$ is strong-acceptable w.r.t. $S$.

In the $E A F$ of Figure [6, the only strong-addmissible sets are \{\}$,\{\mathcal{D}\}$, and $\{\mathcal{D}, \mathcal{F}\}$. Note that argument $\mathcal{A}$ is excluded because its only defense against $\mathcal{C}$ is a blockade-based one.

Example 4. Consider the EAF of Figure [7. The sets $\{\mathcal{B}, \mathcal{D}\}$ and $\{\mathcal{A}, \mathcal{C}\}$ are admissible, but only $\{\mathcal{B}, \mathcal{D}\}$ is strong-admissible. This is because $\mathcal{C}$ is not strongacceptable with respect to $\{\mathcal{A}\}$. The set $\{\mathcal{B}, \mathcal{D}\}$ is also $\mathrm{x}$-admissible, as $\mathcal{B}$ is acceptable with respect to $\{\mathcal{D}\}$ and $\mathcal{D}$ is acceptable with respect to $\{\mathcal{B}\}$.

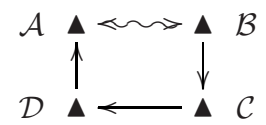

Fig. 7. The set $\{\mathcal{B}, \mathcal{D}\}$ is strong-admissible

Strong-admissibility is a more skeptical criterion for argument acceptance than classical admissible sets. Clearly, an admissible set may be not strong admissible, as in previous examples. Also an x-admissible set is not necessarily strongadmissible: in the framework of Figure 4 the set $\{\mathcal{A}, \mathcal{C}\}$ is x-admissible, but no proper defeat is present and therefore it is not strong-admissible.

Proposition 1. If a set $S$ is strong-admissible, then it is admissible and $x$-admissible.

Proof. Let $S$ be a strong-admissible set. If an argument $\mathcal{A}$ is strong-acceptable with respect to a set $S$, then it is acceptable with respect to $S$, as a collective 
defense is achieved. Thus, every argument in $S$ is acceptable w.r.t $S$, and then $S$ is admissible. Even more, no argument needs its own defense: every argument $\mathcal{X} \in S$ is confirmed by an argument $\mathcal{Y} \in S$. As no argument can be confirmed by itself, then $\mathcal{X} \neq \mathcal{Y}$ and then $\mathcal{X}$ is acceptable with respect to $S-\{\mathcal{X}\}$. Thus, $S$ is x-admissible.

A strong admissible set is perhaps the most reliable defense that an argument may acquire, as it is based only on strictly preferred arguments. It may be considered that an argument in a strong-admissible set is safely justified. However, it is not always possible to count with this kind of defense. In the framework $\Phi_{s k i}$ the only strong-admissible set is the empty set. In the framework of Figure 6 argument $\mathcal{A}$ is somehow defended, but it is rejected according to strongadmissibility. Sometimes it is desirable to adopt a more credulous position of acceptance. Therefore, it is interesting to evaluate the quality of a defense and its alternatives when incomparable or equivalent-in-force defenders are involved. This is addressed in the following section.

\section{$5 \quad$ Weighing Up Defenses}

An argument $\mathcal{A}$ may be collectively defended by several sets of arguments. In $\Phi_{s k i}$, argument $N S$ is defended by $\left\{N S_{1}\right\}$ and it is also defended by $\left\{N S_{2}\right\}$. As a consequence, the union of these sets constitutes a defense for $\mathcal{A}$. The final set of accepted arguments depends on the position adopted by the rational agent, for which the knowledge is modeled by the framework . Sometimes, maximality is required, a position in which an agent accepts all it can defend. Another interesting position is to accept a small set of arguments and its strongest defenders. This corresponds to an agent that, given a special situation where arguments are defended in different manners, decides to accept a minimal set where, if not needed, weakest defenders are discarded. In particular, in extended abstract frameworks, defense can occur with different levels of strength, and when a lot of equivalent-in-force or incomparable arguments are involved in the defeat scenario (leading to several extensions) it is interesting to target the construction of valid extensions according to the appropriate defense.

This evaluation of defenses can be achieved by considering a rational, implicit ordering of argument preferences. This is a common sense property: given two arguments for which a preference must be made, the best scenario is where one of them is effectively preferred to the other. If this is not the case, then at least is desirable to acknowledge the equivalence in strength, so it is clear that both arguments are somehow related. The worst case is to realize that both arguments are not related enough even to evaluate a difference in force. This is formalized in the next definition.

Definition 11. Let $\Phi=\langle A R, \sqsubseteq, \mathbf{C}, \mathbf{R}\rangle$ be an $E A F$. Let $\mathcal{A}$ and $\mathcal{B}$ be two arguments in $A R$ The function pref $: A R \times A R \rightarrow\{0,1,2\}$ is defined as follows

$$
\operatorname{pref}(\mathcal{A}, \mathcal{B})= \begin{cases}0 & \text { if } \mathcal{A} \bowtie \mathcal{B} \\ 1 & \text { if } \mathcal{A} \equiv \mathcal{B} \\ 2 & \text { if } \mathcal{A} \succ \mathcal{B}\end{cases}
$$


The simple graduation of preference conclusions stated in Definition [1] allows the comparison of individual defenses. For an argument $\mathcal{A}$, the strength of its defenders is evaluated as stated in Definition 12 .

Definition 12. Let $\Phi=\langle A R, \sqsubseteq, \mathbf{C}, \mathbf{R}\rangle$ be an $E A F$. Let $\mathcal{A} \in A R$ be an argument with defeater $\mathcal{B}$, which is defeated, in turn, by arguments $\mathcal{C}$ and $\mathcal{D}$. Then

1. $\mathcal{C}$ and $\mathcal{D}$ are equivalent in strength defenders of $\mathcal{A}$ if pref $(\mathcal{C}, \mathcal{B})=\operatorname{pref}(\mathcal{C}, \mathcal{D})$.

2. $\mathcal{C}$ is a stronger defender than $\mathcal{D}$ if $\operatorname{pre} f(\mathcal{C}, \mathcal{B})>\operatorname{pre} f(\mathcal{C}, \mathcal{D})$. It is also said that $\mathcal{D}$ is a weaker defender than $\mathcal{C}$

In the framework $\Phi_{s k i}$, argument $N S_{2}$ is a stronger defender of $N S$ than $N S_{3}$. In the framework of Figure 6 , for argument $\mathcal{A}$, the argument $\mathcal{F}$ is a weaker defender than $\mathcal{D}$ and also than $\mathcal{E}$. The evaluation of a collective defense follows from Definition 12

Definition 13. Let $\Phi=\langle A R, \sqsubseteq, \mathbf{C}, \mathbf{R}\rangle$ be an $E A F$. Let $\mathcal{A} \in A R$ be an argument acceptable with respect to $S_{1} \subseteq A R$. A set of arguments $S_{2} \subseteq A R$ is said to be a stronger collective defense of $\mathcal{A}$ if $\mathcal{A}$ is acceptable with respect to $S_{2}$, and

1. There does not exists two argument $\mathcal{X} \in S_{1}$ and $\mathcal{Y} \in S_{2}$ such that $\mathcal{X}$ constitutes a stronger defense than $\mathcal{Y}$

2. For at least one defender $\mathcal{X} \in S_{1}$ of $\mathcal{A}$, there exists an argument $\mathcal{Y} \in S_{2}$ which constitutes a stronger defense of $\mathcal{A}$.

A set of arguments $S_{2}$ is a stronger collective defense of $\mathcal{A}$ than the set $S_{1}$ if the force of defense achieved by elements in $S_{2}$ is equal or stronger than those in $S_{1}$. Informally, every argument $\mathcal{X}$ in $S_{1}$ has a competitor $\mathcal{Y}$ in $S_{2}$ that is a stronger or equivalent in strength defender. Note that it is possible that $\mathcal{X}=\mathcal{Y}$.

In the framework $\Phi_{s k i}$, the set $\left\{N S_{2}\right\}$ is a stronger defense than $\left\{N S_{3}\right\}$. The set $\left\{N S_{2}, N S_{3}\right\}$ is not considered a stronger collective defense than $\left\{N S_{2}\right\}$ (maximality is not relevant under this notion). On the other hand, $\left\{N S_{2}, N S_{3}\right\}$ is considered a stronger collective defense than $\left\{N S_{3}\right\}$, because of the inclusion of a stronger defender.

The strength of a defense is a pathway to evaluate admissible sets. In Dung's classic abstract framework, admissible sets may be compared, for instance, by set inclusion, and then maximal extensions are of interest. Even then, two admissible sets with the same number of arguments may be considered indistinguishable alternatives for acceptance, as in the Nixon Diamond example [1]. In extended abstract frameworks, defeat may occur in different ways, according to preference criterion $\mathbf{R}$, and this can be used to evaluate the inner composition of an admissible set.

Example 5. Consider the EAF of Figure 8. The admissible sets are $\emptyset$ (trivial), every singleton set, $\{\mathcal{A}, \mathcal{D}\}$ and $\{\mathcal{A}, \mathcal{C}\}$. Argument $\mathcal{A}$ is defended by sets $\{\mathcal{D}\}$ and $\{\mathcal{C}\}$, but the first one is a stronger collective defense than the second one. Then $\{\mathcal{A}, \mathcal{D}\}$ is an admissible set with stronger inner defenses than $\{\mathcal{A}, \mathcal{C}\}$. 


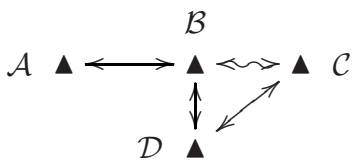

Fig. 8. $\{\mathcal{D}\}$ is a stronger defense of $\mathcal{A}$ than $\{\mathcal{C}\}$

Proposition 2. Let $\langle A R$, $, \mathbf{C}, \mathbf{R}\rangle$ be an $E A F$. If an argument $\mathcal{A} \in A R$ is strong-acceptable with respect to a set of arguments $S \subseteq A R$, then no other set $S^{\prime} \subseteq A R$ constitutes a stronger collective defense than $S$.

Proof. Trivial, as every individual defense cannot be strengthened by another argument because it is achieved by proper defeaters.

Definition 14. An admissible set of arguments $S$ is said to be top-admissible if, for any argument $\mathcal{A} \in S$, no other admissible set $S^{\prime}$ provides a stronger defense of $\mathcal{A}$ than $S$.

In Figure 6, the set $\{\mathcal{A}, \mathcal{D}, \mathcal{E}\}$ is top-admissible, while the set $\{\mathcal{A}, \mathcal{D}, \mathcal{F}\}$ is not. In the framework $A F_{s k i}$, the admissible set $\left\{N S, N S_{1}\right\}$ is top-admissible. The set $\left\{N S, N S_{2}\right\}$ is not, as $N S$ can be benefited from a better defense. In the EAF of Figure 8 , the set $\{\mathcal{A}, \mathcal{D}\}$ is top-admissible. Top-admissibility is also a measure of strength, other than cardinality of admissible sets. Although every maximal (with respect to set inclusion) admissible set is top-admissible, the converse is not true. In Figure 6, the set $\{\mathcal{A}, \mathcal{D}, \mathcal{F}\}$ is top-admissible, but not maximal.

Definition 14 is a measure of quality. What top-admissibility semantics requires is that best admissible sets are selected, according to the strength of every defense. Note that every strong-admissible set is top-admissible, but a topadmissible set may not be strong-admissible as the former is allowing blockadebased defenses.

\section{Conclusions and Related Work}

In this work, new restricted notions of admissibility were introduced by taking into account the strength of an argument defense in extended abstract frameworks 89. . Each of the new admissibility notions presented relies on the presence of blocking defeaters in the argument scenario. A set $S$ is x-admissible if no blocked argument in $S$ needs its own defense. A set is strong-admissible if every argument in that set is defended by arguments strictly preferred to its defeaters, discarding any blockade-based defense. A set is top-admissible if no inner defense can be individually strengthened. As said before, the final set of accepted arguments depends on the position adopted by the rational agent. The aim of this work was to provide new views for selecting an admissible set of arguments in extended abstract frameworks. 
Acceptability notions are defined in [1], and many interesting works are based on these concepts. Other extensions based on acceptability of arguments were defined, although using classic frameworks, and then usually maximal or minimal sets are relevant. In [10] minimality is explored as a property of defenses by distinguishing two types of arguments, one of them mandatory as a defense, while the other used only when is needed. Thus, the defense is also weighed out according to relevance of defenders. Despite the framework used, weaker defenders in EAF may be considered as a form of restricted arguments in [10].

Preferences between arguments are also present in other works, notably in 4, where the notion of strict-defense influenced strong-acceptability in EAF. Also in that work an argument may defend itself, although in a different way: it is preferable to its attackers. In $E A F$ self-defended arguments are related to symmetric blocking and each argument's context. Preference is used in [6], where value-based argumentation frameworks are presented. In that work, defeat is derived by comparing arguments. Similar to [4], an attack is overruled (i.e. it does not succeed) if the attacked argument is preferred to its attacker. Otherwise, a defeat relation arises, even when both argument may promote equal or unrelated values. In $E A F$ this leads to blocking defeat, the basis for distinguishing grades on defense, as it is done in the present work. In [11] principles for the evaluation of extensions are presented; skepticism among them. According to the weak skepticism relation, a strong-admissible set is more skeptical than admissible sets. Also top-admissibility is more skeptical than classical admissibility.

\section{References}

1. Dung, P.M.: On the Acceptability of Arguments and its Fundamental Role in Nomonotonic Reasoning and Logic Programming. In: Proc. of the 13th. IJCAI 1993, pp. 852-857 (1993)

2. Vreeswijk, G.A.W.: Abstract argumentation systems. Artificial Intelligence 90(12), 225-279 (1997)

3. Amgoud, L., Cayrol, C.: On the acceptability of arguments in preference-based argumentation. In: UAI 1998. 14th Conference on Uncertainty in Artificial Intelligence, pp. 1-7. Morgan Kaufmann, San Francisco (1998)

4. Amgoud, L., Cayrol, C.: A reasoning model based on the production of acceptable arguments. Annals of Mathematics and Artificial Intelligence 34(1-3), 197-215 (2002)

5. Amgoud, L., Perrussel, L.: Arguments and Contextual Preferences. In: CD 2000. Computational Dialectics-Ecai workshop, Berlin (2000)

6. Bench-Capon, T.: Value-based argumentation frameworks. In: Proc. of Nonmonotonic Reasoning, pp. 444-453 (2002)

7. Martínez, D., García, A., Simari, G.: Progressive defeat paths in abstract argumentation frameworks. In: Proceedings of the 19th Conf. of the Canadian Society for Computational Studies of Intelligence 2006, pp. 242-253 (2006)

8. Martínez, D., García, A., Simari, G.: On acceptability in abstract argumentation frameworks with an extended defeat relation. In: COMMA 2006. Proc. of I. Intl. Conf. on Computational Models of Arguments, pp. 273-278 (2006) 
9. Martínez, D., García, A., Simari, G.: Modelling well-structured argumentation lines. In: Proc. of XX IJCAI-2007, pp. 465-470 (2007)

10. Cayrol, C., Doutre, S., Lagasquie-Schiex, M.C., Mengin, J.: "minimal defence": a refinement of the preferred semantics for argumentation frameworks. In: NMR, pp. 408-415 (2002)

11. Baroni, P., Giacomin, M.: Evaluation and comparison criteria for extension-based argumentation semantics. In: COMMA 2006. Proc. of I. International Conf. on Computational Models of Arguments, pp. 157-168 (2006) 